hep-th/0109058

UT-KOMABA/01-05

\title{
World-Sheet String Duality and the Hidden Supersymmetry ${ }^{\|}$
}

\author{
TAMIAKI YONEYA I] \\ Institute of Physics, University of Tokyo \\ Komaba, Meguro-ku, 153 Tokyo
}

\begin{abstract}
It is reviewed how space-time supersymmetry is realized nonlinearly in open superstring theory without making the GSO projection. We show that the world-sheet string dualities, viz. dualities of open-closed strings and of open-open strings, play crucial roles for the existence of 10 dimensional $N=2$ supersymmetry in a spontaneously broken phase. We also speculate on a possible mechanism of the restoration of supersymmetry from the viewpoint of world-sheet dynamics.
\end{abstract}

\section{Introduction}

One of the interesting new concepts established in recent advances of superstring theory is the notion of unstable D-branes [1]. In a sense, unstable D-branes may be viewed as more fundamental than stable (BPS) D-branes, since stable D-branes can be classified using K-theory starting from the systems of unstable D9 (and/or anti-D9) branes in type IIA(B) theories [2]. Recent investigations in noncommutative field theory and also in string field theory suggest further that all D-branes may be understood even dynamically on the basis of such unstable systems. However, most of such dynamical discussions have so far been restricted to bosonic strings. In particular, the role of space-time supersymmetry (susy) in unstable systems has not been understood appropriately. The space-time

* Talk presented at the 10th International Symposium on String Theory (July 3-7, Fukuoka, Japan, 2001) and a part of lectures given at the Summer Conference on Strings (July 16-27, Beijing, China, 2001).

$\dagger$ e-mail address: tam@hep1.c.u-tokyo.ac.jp 
supersymmetry is of course broken in such unstable vacua. Nonetheless its existence in a hidden (spontaneously broken) form must be important, since the spontaneously broken supersymmetry can in principle constrain the dynamics in some definite manner.

In the present talk, I would like to review, on the basis of two previous works [3] [4], the status of the above question. Throughout the discussions below, I will mostly emphasize the standpoint of world-sheet dynamics, although string field theory is in general potentially suitable for investigating spontaneously broken symmetries. The main reason for this is that the world-sheet string dualities turn out to be crucial for the consistent implementation of hidden susy for nonzero $\alpha^{\prime}$, while string field theory is not, unfortunately at least in its present level of development, convenient for exhibiting such stringy characteristics.

\section{Evidence of hidden supersymmetry}

Questions which we should ask first may be those: How can we see the spontaneouly broken susy in terms of perturbative theory of the open strings describing unstable Dbranes? Are there any concrete signatures of the broken susy? A possible clue in answering such questions is that unstable D-branes should be coupled to gravity or closed strings consistently at least in the sense of perturbation theory. One among the most intriguing phenomena in string theory is that open-string loop diagrams are dual to closed-string tree diagrams. This implies that open-string loop amplitudes themselves without direct coupling to closed strings must exhibit certain trace of the type II supersymmetry of closed strings, to the extent that they are acceptable as perturbative amplitudes.

Let us take the simplest example of a single D9-brane in type IIA theory and consider the one-loop partition function of a single open string in the NSR formalism, which is, ignoring the momentum part and fixing the moduli parameter $\tau$,

$$
Z(\tau)=\operatorname{Tr}_{N S}\left(q^{2 N_{N S}-1}\right)-\operatorname{Tr}_{R}\left(q^{2 N_{R}}\right)
$$

where $q=\mathrm{e}^{-\tau / 2 \alpha^{\prime}}$ and $N_{N S}, N_{R}$ are the level operators for the NS sector and R sector, respectively. Note that we do not here make the standard GSO projection. Then the partition function has nonzero contribution only from the oppositely GSO projected sector as

$$
Z(\tau)=\frac{1}{\prod_{n=1}^{\infty}\left(1-q^{2 n}\right)^{8}}\left(\frac{1}{2 q} \prod_{n=1}^{\infty}\left(1+q^{2 n-1}\right)^{8}+\frac{1}{2 q} \prod_{n=1}^{\infty}\left(1-q^{2 n-1}\right)^{8}-\frac{1}{2} 16 \prod_{n=1}^{\infty}\left(1+q^{2 n}\right)^{8}\right) .
$$

In the case of the ordinary GSO projected sector, the sign in front of the second term inside the large round brace is opposite, and hence $Z(\tau)$ vanishes due to the well known 
Jacobi's 'abstruse' identity. This vanishing is a consequence of the matching of the degrees of freedom between bosons and fermions at each fixed mass level. Conversely, the nonvanishing result of the partition function (2.2) means that there is no matching of the degrees of freedom between bosons and fermions at fixed mass levels.

However, if supersymmetry is hidden, there must be the similar matching with respect to the total degrees of freedom. Namely, the number of the degrees of freedom must be equal between bosons and fermions when we ignore the difference of masses. Furthermore, in the case of ordinary local field theories, it is well known that spontaneously broken supersymmetry usually leads to the following mass formula for the linearized excitation levels:

$$
\operatorname{Tr}\left((-1)^{F} M^{2}\right)=0
$$

where $F$ is the space-time fermion number. In the present case, this is not well defined as it stands since there are an infinite number of states with increasing masses. However, the partition function can be used to obtain a general (regularized) mass formula by

$$
A_{n} \equiv \lim _{\tau \rightarrow 0} \operatorname{Tr}\left((-1)^{F} M^{2 n} \mathrm{e}^{-\tau M^{2}}\right)=(-1)^{n} \lim _{\tau \rightarrow 0} \frac{d^{n}}{d \tau^{n}} Z(\tau)
$$

This implies that the whole information on the number of degrees of freedom and on the mass formula of open-string spectrum as well can equivalently be expressed in terms of the low-energy spectrum of closed strings in the dual channel, since the short-time limit $\tau \rightarrow 0$ in the open-string channel is equivalent to the large time $1 / \tau \rightarrow \infty$ propagation of closed strings $\left(\sigma=\tau / 2 \pi \alpha^{\prime}\right)$ :

$$
Z(\tau) \rightarrow \frac{\sigma^{4}}{2}\left(16+16+(256+256) \mathrm{e}^{-2 \pi / \sigma}+O\left(\mathrm{e}^{-4 \pi / \sigma}\right)\right)
$$

which leads to

$$
A_{n}=0, \quad(n \neq 4) .
$$

Thus, not only the total degrees of freedom match between (space-time) bosons and fermions, but also the quantities (mass) ${ }^{2 n}$ averaged over the whole open-string degrees of freedom are equal between bosons and fermions for all $n$ except for $n=4$. It is more than natural to imagine the existence of supersymmetry behind this surprising cancellations. Since the above duality is a consequence of the interplay of the whole tower of open string states, we expect that the exact hidden supersymmetry can only be properly explained by taking account of the whole massive higher excitations of open strings, at least for nonzero $\alpha^{\prime}$. It should be emphasized that the consistency of the above interpretation is owing to the fact that the closed string channel is automatically restricted to the ordinary GSO projected sector. Otherwise, we would have tachyon in the closed-string channel which completely ruins the vanishing of $A_{n}$ because of the divergence in the limit $\tau \rightarrow 0$. In the 
open-string language, the GSO projection for closed strings comes about by the insertion of the factor $(-1)^{F}$ in the partition function. This restricts the excitation of fermionic world-sheet fields to be odd in both left and right moving fields in the closed-string sector, irrespectively of NS-NS or of R-R sector. This is nothing but the GSO projection of closed strings.

Here we have treated the simplest case of a single D9-brane in type IIA theory. Extensions to multiple D9 branes and to D9- $\overline{\mathrm{D} 9}$ in type IIB theory is straightforward, since the above open-closed string duality is valid separately for both the sectors of ordinary GSO and opposite GSO projections. Recall that the open strings stretching between D9 and anti-D9 brane are oppositely GSO projected. All we have to be careful is to avoid the Ramond-Ramond anomaly. For example, in the case of type IIB theory, we have to consider the same number of D9 and anti-D9 branes. This will ensure that the hidden susy for type IIB case is the chiral $N=2$ susy.

In the zero-slope limit $\alpha^{\prime} \rightarrow 0$, we expect that the consistent coupling of massless open string states with supergravity fields is guaranteed again by the open-closed string duality. For the resulting low-energy effective theory to be consistent, the massless open-string sector must be described by an appropriate nonlinear realization of $N=2$ supersymmetry. The latter can be obtained by a systematic $\alpha^{\prime}$ expansion of the full theory, as we will discuss later. This amounts to eliminating all the massive modes, including the tachyon, in terms of massless modes using the equations of motion. Once we include the tachyon of the open-string sector, however, we must take the other massive excitations into account on equal footing with the tachyon. In principle, it is possible to derive some effective theory expressed in terms of massless open string states and only tachyon by eliminating the other massive excitations through the equation of motion. However, it seems very hard to recast such a theory into closed and useful form.f

Another interesting class of total spontaneous breaking of supersymmetry is obtained when the supersymmetry is broken by an orientifold plane, being accompanied by the suitable number (32) of $\overline{D 9}$ branes [6]. It was confirmed [7] that the above observation applies to this case as well. Including this case, an extensive discussion on the consistency (viz. anomaly cancellation) of various models with spontaneous broken supersymmetry was given in [8].

\section{Massless Ramond fermion as Nambu-Goldstino}

Let us next study another important trace of spontaneously broken supersymmetry. If we first switch off gravity and consider open strings propagating in flat space-time, there

\footnotetext{
$\ddagger$ Such a possibility was touched upon briefly in [3] to motivate discussions on the hidde susy and was further studied more systematically in [5].
} 
must be the Nambu-Goldstone state (Goldstino, for brevity) associated with the global supersymmetry of the open-string sector. In the case of type II theories, we should have two corresponding Goldstino excitations in 10 dimensions. Such Goldstinos would be 'eaten' by gravitinos through super Higgs effect when we take the mixing with closedstring states into account. Nevertheless, in perturbation theory, it is meaningful and useful to study Goldstino as the crucial signature of spontaneous breaking of supersymmetry in the open-string sector. Let us therefore consider whether and how the massless fermionic ground states of the open fermionic strings can be regarded as the Goldstino.

In the field-theory language, the spontaneous symmetry breaking is in general reflected to the appearance of inhomogeneous terms in the corresponding symmetry transformation law of the Goldstone field $\psi$,

$$
\delta \psi=\epsilon+\text { linear and higher terms, }
$$

or equivalently, to the presence of linear terms

$$
j^{\mu}=\gamma^{\mu} \psi+\text { quadratic and higher terms, }
$$

in the corresponding conserved current. In the first quantized world-sheet picture, both of these features are summarized into the following property of the world-sheet (super)current: The conservation of the world-sheet charge is violated, but the violation is compensated by the emission or absorption of the Goldstino from the vacuum at zero momentum. More concretely, the divergence of the world sheet (super)current must be proportional to the zero-momentum limit of the vertex operator of the massless Goldstino state. The violation of world-sheet charge conservation occurs because the first quantized world-sheet supercurrent only represents the effect of the quadratic terms of the spacetime current. On the other hand, the compensation of charge nonconservation due to the Goldstino emission at zero momentum corresponds to the shift $\delta \psi$ of the Goldstino field, which is represented by insertion of the zero-momentum vertex operator and corresponds to the action generated by the linear term in the space-time charge associated to the linear term of the conserved space-time current. Actually, the massless gauge bosons, including graviton and its partners, can all be regarded as Goldstone excitations in similar manner, corresponding to globally nontrivial gauge transformations 3 which are broken in the ordinary perturbative vacua.

It is easy to check that, without the ordinary GSO projection, the world-sheet supercurrent indeed satisfies the above criteria. First, in the NSR formalism, the world-sheet

$\S$ The simplest example of this is the $U(1)$ gauge transformation $\delta A_{\mu}(x)=\partial_{\mu} \lambda(x)$ with $\lambda(x)=c_{\mu} x^{\mu}$ with $c_{\mu}$ being a constant vector. 
current $s_{\alpha}^{a} \quad\left(\partial_{a} s_{\alpha}^{a}=0\right)$ is given as

$$
\begin{aligned}
& s_{\alpha}^{0}(\tau, \sigma)=\frac{1}{2}\left(S_{\alpha} \mathrm{e}^{-\phi / 2}(\tau-\sigma)+S_{\alpha} \mathrm{e}^{-\phi / 2}(\tau+\sigma)\right), \\
& s_{\alpha}^{1}(\tau, \sigma)=\frac{1}{2}\left(S_{\alpha} \mathrm{e}^{-\phi / 2}(\tau-\sigma)-S_{\alpha} \mathrm{e}^{-\phi / 2}(\tau+\sigma)\right),
\end{aligned}
$$

in terms of the spin operator $S_{\alpha}(\tau \pm \sigma)$ ( $\alpha=32$ component spinor index in 10 dimensions) and bosonized ghost $\phi(\tau \pm \sigma)$. In the presence of oppositely GSO projected states, the conservation of the world-sheet charge

$$
Q_{\alpha}(\tau)=\int_{0}^{\pi} d \sigma s_{\alpha}^{0}(\tau, \sigma)
$$

is in general violated at the boundary of open strings:

$$
\begin{gathered}
\frac{d Q_{\alpha}(\tau)}{d \tau}=-\int_{0}^{\pi} d \sigma \partial_{1} s_{\alpha}^{1}(\tau, \sigma)=-S_{\alpha} \mathrm{e}^{-\phi / 2}(\tau-\pi)+S_{\alpha} \mathrm{e}^{-\phi / 2}(\tau+\pi) \\
\Downarrow \\
\frac{d Q_{\alpha}(\tau)}{d \tau}=-2 S_{\alpha} \mathrm{e}^{-\phi / 2}(\tau-\pi),
\end{gathered}
$$

since the spin operator can be double-valued in the sense [7]

$$
S(\tau)=-S(\tau-2 \pi)
$$

In the case of closed-string vertex operators, such double-valuedness is not acceptable, since it introduces nonlocality in the world-sheet field theory. In the case of open strings, on the other hand, this is allowed, as long as the double-valued operators live only at the boundary. The right hand side of (3.7) coincides with the vertex operator (of zero momentum) of the massless Ramond ground state. Thus, we can indeed identify the (singlet) Ramond ground state as the Goldstino state corresponding to the spontaneous broken $N=2$ susy: $N=2$ since there are $32(=16+16)$ components of the massless Goldstino fields.

At a very formal level, the above structure can in fact be translated into the following transformation law of string fields in the framework of Witten's open superstring field theory:

$$
\begin{aligned}
\delta a & =\epsilon^{\alpha} Q_{\alpha} \psi, \\
\delta \psi & =\epsilon^{\alpha} Q_{\alpha} Q_{L} \mathcal{I}+X\left(\frac{\pi}{2}\right) \epsilon^{\alpha} Q_{\alpha} a,
\end{aligned}
$$

where $a$ and $\psi$ are the bosonic and fermionic parts, respectively of the string fields. Other notations are as follows: $I=$ identity field, $Q_{L}=$ half-integrated BRST operator, $X\left(\frac{\pi}{2}\right)$

\footnotetext{
ब In the Euclidean metric on the world-sheet, this is $S(z)=-S\left(e^{i 2 \pi} z\right)$.
} 
$=$ picture changing operator inserted at the mid point $\sigma=\pi / 2$. For more details, see [3] and the references therein. The first term of the right hand side of (3.9) corresponds to the Goldstino nature of the string field. Unfortunately, however, it is not clear whether this transformation law can be justified rigorously, because of the well known problems related to the midpoint anomalies of superstring field theory. Berkovitz [9] proposed an interesting new formulation in which the mid-point insertion of picture changing operator is avoided. It is desirable to extend the above susy transformation law to this formulation.

The same conclusion as above can be reached by using the Green-Schwarz formalism as well. To see this, it is useful to reconsider the partition function (2.2) again. Although this is nonvanishing, it can be rewritten by using the Jacobi identity as

$$
\frac{1}{q} \frac{\prod_{n=1}^{\infty}\left(1-q^{2 n-1}\right)^{8}}{\prod_{n=1}^{\infty}\left(1-q^{2 n}\right)^{8}} .
$$

This result coincides precisely with the spectrum of the Green-Schwarz open string when we assume that the world-sheet spinor fields $S_{\alpha i}(i=1,2)$ (which transform as space-time spinor, $\alpha$ being the spinor index) satisfy the 'anti-periodic' boundary condition as

$$
S_{\alpha 1}(\tau, 0)=-S_{\alpha 2}(\tau, 0), \quad S_{\alpha 1}(\tau, \pi)=S_{\alpha 2}(\tau, \pi),
$$

where the two-component index $i$ denotes the left and right moving components of the world-sheet spinor fields. Recall that in the case of the standard periodic boundary condition we would have the same $+($ or -$)$ sign at both end points $\sigma=0, \pi$ in (3.10). The above boundary condition leads to the mass operator

$$
\alpha^{\prime} M_{G S-}^{2}=\sum_{n=1}^{\infty} \alpha_{-n}^{i} \alpha_{n}^{i}+\sum_{r=1 / 2}^{\infty} r S_{-r A} S_{r A}-\frac{1}{2},
$$

giving directly the above form of the partition function. Thus, we have established that in the GS formalism, two possible boundary conditions, periodic and anti-periodic, of the spinor fields just correspond to the ordinary GSO projected and oppositely GSO projected sectors, respectively, of the NSR formalism. In this language, the fact that the closed string channel is automatically GSO projected is manifest, since the insertion of the factor $(-1)^{F}$ makes the boundary condition for the spinor field in the closed string channel to be periodic, irrespectively of boundary conditions in the open string channel.

It is not difficult to check that the same mechanism which enables us to identify the massless fermion states with the Goldstinos corresponding to the spontaneous breaking of $N=2$ supersymmetry as that in the NSR formalism works in this formalism as well. Namely, the world-sheet supercharge is not conserved when we include the antiperiodic spinors. But the violation is compensated by the insertion of massless fermion vertex operators at open string boundaries. For details about this and more, I would like to invite the reader to the original paper [3]. 


\section{Effective low-energy theory of Goldstino}

We next briefly discuss the effective low-energy description of the open-string Goldstinos. One of the characteristic features of Goldstone excitations in general is that the lowenergy behavior of their scattering is constrained by various low-energy theorems reflecting nonlinear symmetry transformation law. In particular, a 4-point amplitude of them must vanish as the external momenta approach to zero, since the effective lagrangian must necessarily contain derivatives to preserve the symmetry under the transformation $\delta \psi=$ $\epsilon+\cdots$. In the present case, there arises a small puzzle related to this: Since there exists a scalar tachyon $\phi$ which couples to massless fermion fields $\psi_{ \pm}$, the indices \pm being the chirality, we would naively expect the appearance of the contact term

$$
\bar{\psi}_{+} \psi_{-} \bar{\psi}_{+} \psi_{-}
$$

in the zero-momentum limit, which is mediated by the exchange of the scalar tachyon through Yukawa interaction $\phi \bar{\psi}_{+} \psi_{-}$. The generic four-fermion string amplitudes indeed exhibit a pole corresponding to tachyon in the open-string channels where the opposite GSO sector can propagate, and its residue is consistent with Yukawa coupling. But this contact term must be cancelled to be consistent with our interpretation of the massless fermion as Goldstino. It would be a quite nontrivial phenomenon, since only possibility of such cancellation is due to the contribution of higher massive modes.

Let us first derive the effective lagrangian for fermion scattering. A convenient way of doing this is to start from the generalized DBI action [10 11] (or we should rather call Volkov-Akulov type action) of a space filling (nonBPS) D9 brane.

$$
S_{\mathrm{eff}}=-T \int d^{10} x \sqrt{-\operatorname{det} G_{\mu \nu}}
$$

where

$$
\begin{gathered}
G_{\mu \nu}=\eta_{\mu \nu}+\lambda F_{\mu \nu}+\lambda^{2} S_{\mu \nu}^{(2)}+\lambda^{4} S_{\mu \nu}^{(4)}, \\
S_{\mu \nu}^{(2)}=-i \bar{\psi}_{+} \Gamma_{\nu} \partial_{\mu} \psi_{+}-i \bar{\psi}_{-} \Gamma_{\mu} \partial_{\nu} \psi_{-}, \\
S_{\mu \nu}^{(4)}=-\frac{1}{4}\left(\bar{\psi}_{+} \Gamma^{\alpha} \partial_{\mu} \psi_{+} \bar{\psi} \Gamma_{\alpha} \partial_{\nu} \psi+\bar{\psi}_{-} \Gamma^{\alpha} \partial_{\nu} \psi_{-} \bar{\psi} \Gamma_{\alpha} \partial_{\mu} \psi\right) .
\end{gathered}
$$

The $(N=2)$ susy transformation law $\left(\psi \equiv \psi_{+} \oplus \psi_{-}\right)$is

$$
\begin{gathered}
\delta \psi=\frac{1}{\lambda} \epsilon-i \frac{\lambda}{2}\left(\bar{\epsilon} \Gamma^{\mu} \psi\right) \partial_{\mu} \psi \\
\delta A_{\mu}=\frac{i}{2} \bar{\epsilon} \Gamma_{11} \Gamma_{\mu} \psi+\frac{\lambda^{2}}{24}\left(\bar{\epsilon} \Gamma_{11} \Gamma_{\nu} \psi \bar{\psi} \Gamma^{\nu} \partial_{\mu} \psi+\bar{\epsilon} \Gamma_{\nu} \psi \bar{\psi} \Gamma_{11} \Gamma^{\nu} \partial_{\mu} \psi\right) \\
-i \frac{\lambda}{2}\left(\bar{\epsilon} \Gamma^{\nu} \psi\right) \partial_{\nu} A_{\mu}-i \frac{\lambda}{2}\left(\bar{\epsilon} \Gamma^{\nu} \partial_{\mu} \psi\right) A_{\nu}
\end{gathered}
$$


Comparing with the usual linear transformation law of super Yang-Mills theory, this form is a bit strange, since the transformation of $\psi$ does not contain any linear term mixing $\psi$ and $A_{\mu}$. However, this is an artifact of the choice of fields. By performing a field redefinition appropriately in such a way that there remains no cubic interaction terms in the action, we can rewrite the susy transformation law as

$$
\delta \psi \equiv \sum_{n=-1} \lambda^{n} \delta \psi^{(n)}, \quad \delta A_{\mu} \equiv \sum_{n=0} \lambda^{n} \delta A_{\mu}^{(n)}
$$

where

$$
\begin{gathered}
\delta \psi_{ \pm}^{(-1)}=\epsilon_{ \pm}, \quad \delta \psi_{ \pm}^{(0)}= \pm \frac{1}{4} \Gamma^{\mu \nu} \epsilon_{ \pm} F_{\mu \nu}, \quad \ldots \\
\delta A_{\mu}^{(0)}=\frac{i}{2} \bar{\epsilon} \Gamma_{11} \Gamma_{\mu} \psi, \quad \ldots
\end{gathered}
$$

In the lowest order with respect to derivatives, this reduces to the standard linear transformation law if we set $\psi_{-}=0$. By making expansion with respect to the power of fermion fields, the resulting effective action for 4 fermion scatterings is

$$
\begin{aligned}
S_{\mathrm{eff}}^{(4 \psi)}=-\frac{\lambda^{2}}{24}\left(\bar{\psi}_{+} \Gamma_{\mu} \partial_{\nu} \psi_{+} \bar{\psi}_{+} \Gamma^{\mu} \partial^{\nu} \psi_{+}+\bar{\psi}_{-}\right. & \left.\Gamma_{\mu} \partial_{\nu} \psi_{-} \bar{\psi}_{-} \Gamma^{\mu} \partial^{\nu} \psi_{-}\right) \\
& -\frac{\lambda^{2}}{4} \bar{\psi}_{+} \Gamma_{\mu} \partial_{\nu} \psi_{+} \bar{\psi}_{-} \Gamma^{\mu} \partial^{\nu} \psi_{-} .
\end{aligned}
$$

Thus, the 4 fermion amplitudes must vanish as the squares of momenta. We have confirmed that the required cancellation indeed occurs between the tachyon exchange and that of higher excitation modes of open string in the oppositely GSO projected sector. For this, the $s$ - $t$ duality symmetry

$$
A_{1+, 3-, 2+, 4-}(s, t)=A_{1+, 4-, 2+, 3-}(t, s)
$$

between the mutually dual planar amplitudes with cyclic ordering $(1+, 3-, 2+, 4-)$ is crucial: This symmetry is in contradiction with the fermi antisymmetry with respect to the exchange $1 \leftrightarrow 2$, and hence this particular ordering does not contribute to the final amplitude. The peculiarity occurring here is related to the apparent violation of spin-statistics theorem in the NSR formalism when both the GSO sectors are included. The zero-momentum limit of this planar amplitude does not vanish, while the other planar contributions with orderings such as $(1+, 2+, 3-, 4-)$ contribute, but do vanish in the zero-momentum limit. Combining all these properties, it turns out that the net results for the low-energy behavior of the total string amplitudes agree precisely with the prediction of the above low-energy effective action. The indispensable role of the higher excited states and open-open string duality is consistent with our previous observation on the importance of the open-closed string duality. For the fuller account of the above remarkable properties of Goldstino scatterings in superstring theory, I would like to refer the reader to our paper [4]. 


\section{World-sheet mechanism for susy restoration?}

As the final topic of this talk, I would like to speculate on the possible mechanism of restoring supersymmetry from its spontaneously broken phase. In the low-energy approximation of the previous section, the supersymmetry is restored if we have the condensation of fermion bilinears characterized by $\left\langle i \bar{\psi}_{ \pm \beta} \partial_{\mu} \psi_{ \pm \alpha}\right\rangle=-\left(\Gamma_{\mu}\left(1 \pm \Gamma_{11}\right) / 2\right)_{\alpha \beta} / 5 \lambda^{2}$, in terms of the fermion fields before the field redefinition which cancels the inhomogeneous term of the susy transformation law. If we wish to avoid the low-energy approximation, an obvious approach to this question would be to use string field theory. In fact, there is a trivial way of eliminating the inhomogeneous term in (3.9), namely the shift $a \rightarrow a-Y\left(\frac{\pi}{2}\right) Q_{I} \mathcal{I}$. This completely eliminates the kinetic term and makes the resulting action purely cubic. However, this shift of string field is not yet the condensation of tachyon, since the shifted part actually does not correspond to the oppositely GSO projected sector. To obtain the stable open string vacuum, we would have to further shift the opposite GSO sector by an appropriate classical string field configuration which is annihilated by the supercharge $Q_{\alpha}$.

Actually, even before going to this problem, the trivial shift of this kind raises a puzzle. The value of the action at the classical vacua is zero either before or after the above shift of string field. But, the original action before any shift is supposed to describe an unstable D9 brane. Then, after restoring supersymmetry, the vacuum value of the action must have been lowered by the tension of the unstable D9 brane. This puzzle seems to be indicative of the danger involved in the formal manipulations such as the ones associated with the identity field, midpoint insertion of picture changing operator, and so on. With regard to these difficulties, a promising possibility may be to use the version of superstring theory proposed in [9], as already mentioned before.

Instead of pursuing this problem further within the framework of string field theory, let us turn our discussion to the world-sheet approach. Our intuitions on various dynamical questions such as symmetry breaking and its restoration usually depend on field theory. However, one of the remarkable features of string theory is that physical pictures on various field theoretical phenomena are remarkably simplified often by using rather the naive world-sheet picture. It would be very nice if we have such an example again in the present problem. In fact, the so-called boundary string field approach [12] clearly suggests a simple physical picture that after tachyon condensation only surviving open strings are those with the world sheets whose boundaries shrink to points in target space-time. Then it is natural to regard the open string boundaries as some sort of defects on closed-string world sheets. From such a viewpoint, the restoration of $N=2$ susy could occur when the boundary conditions for the world-sheet spinors were dynamically flipped at the defects such that the left and right movers propagate independently of each other. 
The questions are then how such a flip of boundary condition can occur and how to treat such a phenomenon dynamically. Kinematically, it seems clear that there must be the same number of dynamical degrees before and after the flip at the defect. Suppose that the defect is located between $\sigma=\sigma_{0}$ and $\sigma_{0}+a$, $a$ being the lattice constant. If the defect corresponds to an unstable D-brane or to a D-brane and anti-D brane system, there are open strings with all possible combinations of the boundary conditions,

$$
S_{+}\left(\sigma_{0}+a\right)=0 \quad \text { or } \quad S_{-}\left(\sigma_{0}+a\right)=0
$$

and

$$
S_{+}\left(\sigma_{0}\right)=0 \quad \text { or } \quad S_{-}\left(\sigma_{0}\right)=0,
$$

where we defined new combinations of spinor components $S_{ \pm}=\left(S_{1} \pm S_{2}\right) / 2$. For instance, in the case of D9- $\overline{\mathrm{D} 9}$ system in type IIB theory, we can consistently choose $S_{+}=0$ and $S_{-}=0$ at the boundaries attached to D-brane and anti-D-brane, respectively. The boundary conditions which preserve one half of supersymmetry are those with the same \pm indices (viz. DD and $\overline{\mathrm{DD}}$ strings) at the both boundaries, while the nonsupersymmetric ones (viz. $\mathrm{D} \overline{\mathrm{D}}$ and $\overline{\mathrm{D}} \mathrm{D}$ strings) correspond to the choices of different signs at the two end points. The closed string configuration after the annihilation of the defect on the other hand is represented as $S_{ \pm}\left(\sigma_{0}+a\right)=S_{ \pm}\left(\sigma_{0}\right)$, realizing $N=2$ susy provided that the annihilation of the defect occurred also in the bosonic part. The possibility of dynamically flipping from the open-string boundary conditions to that of closed string arises only when open string excitations with all the above combinations are included, since then appropriate recombinations of spin components can in principle lead to the flip. Contrary to this, in the case of BPS D-branes, either one of $S_{+}$or $S_{-}$vanishes at all times and hence we can never excite the spinor components such that these two components $S_{ \pm}$ become independent propagating modes.

Of course, our argument is very naive and is not sufficient to show that the flip of boundary condition of the desired type can be achieved. For that, we need to develop a systematic formulation of D-branes and fundamental strings such that the creation and annihilation of D-branes and associated change of boundary conditions can be dealt with as dynamical processes on closed-string world sheets. One candidate for such possibilities is supermembrane theory, or matrix-string theory as its regularized version. It is possible to interpret D0 $(\overline{\mathrm{D} 0})$-branes as soliton-like excitations on supermembranes. In connection with this, we have recently proposed [13] a systematic method of directly mapping supermembranes wrapped around a circle to matrix strings in the large $N$ limit. Recall that matrix-string theory is a framework in which both fundamental strings and D-particles can be treated dynamically. I hope that this new development will be useful for discussing various dynamical issues related to the hidden susy of string/M theory and other important aspects of nonperturbative string theory. 
Acknowledgments

I would like to thank N. Berkovitz for collaborative discussions about the possibility of realizing the hidden susy using his approach to superstring field theory. I am also grateful

to Y. Matsuo for stimulating discussions on string field theory during the symposium. The present work is supported in part by Grant-in-Aid for Scientific Research (No. 12440060) from the Ministry of Education, Science and Culture.

\section{References}

[1] A. Sen, JHEP 08 (1998) 010 ; hep-th/9805019; JHEP 08 (1998) 012 ; hep-th/9805170.

[2] E. Witten, JHEP 12 (1998) 019; hep-th/9810188.

P. Hořava, Adv. Theor. Math. Phys. 2 (1999) 1373; hep-th/9812135.

[3] T. Yoneya, Nucl. Phys. B576 (2000) 219; hep-th/9912255.

[4] T. Hara and T. Yoneya, Nucl. Phys. B602(2001) 499; hep-th/0010173.

[5] S. Terashima and T. Uesugi, JHEP 0105(2001)054; hep-th/0104176.

[6] S. Sugimoto, Prog. Theor. Phys. 102(1999) 685; hep-th/9905159. See also, E. Dudas and J Mourad, hep-th/0012071.

[7] S. Moriyama, hep-th/0107203.

[8] J. H. Schwarz and E. Witten, JHEP 0103(2001) 032; hep-th/0103099.

[9] For a recent review on this, see N. Berkovitz, hep-th/0105230 and references therein.

[10] A. Sen, JHEP 10 (1999) 008; hep-th/9909062.

[11] M. Aganagic, C. Popsecu and J. H. Schwarz, Nucl. Phys. B495 (1997) 99:hep-th/9612080.

[12] A. A. Gerasimov and S. L. Shatashvilli, hep-th/0009103.

D. Kutasov, M. Marino and G. Moore, hep-th/0009148.

[13] Y. Sekino and T. Yoneya, hep-th/0108176. 